\title{
Biomarqueurs prédictifs de réponse aux inhibiteurs de points de contrôle immuns
}

\section{Predictive biomarkers of response to immune checkpoint inhibitors}

\author{
Alexandra Frelau ${ }^{1} *$, Marc Pracht ${ }^{1}$, Samuel Le Sourd ${ }^{1}$, Alexandra Lespagnol ${ }^{2}$, \\ Romain Corre $^{3}$, Cédric Ménard ${ }^{4}$, Karin Tarte ${ }^{4}$, Jean Mosser ${ }^{2,5}$, Julien Edeline ${ }^{1,6}$
}

${ }^{1}$ Oncologie médicale - CLCC Eugène Marquis UNICANCER Avenue de la Bataille Flandres-Dunkerque, 35042 Rennes Cedex

${ }^{2}$ Laboratoire de génétique somatique des cancers CHU Rennes, 2 rue Henri Le Guilloux

35033 Rennes cedex 9

${ }^{3}$ Service de pneumologie CHU Rennes, 2 rue Henri Le Guilloux

35033 Rennes cedex 9

${ }^{4}$ Laboratoire d'immunologie, thérapie cellulaire et hématopoïèse INSERM U197 CHU Rennes, 2 rue Henri Le Guilloux 35033 Rennes cedex 9

${ }^{5}$ Institut de génétique et développement IGRD UMR 6290 CNRS - UR1 CHU Rennes, 2 rue Henri Le Guilloux 35033

Rennes cedex 9

${ }^{6}$ Unité de recherche Foie, métabolisme et cancer - UMR991 - CHU Rennes, 2 rue Henri Le Guilloux

35033 Rennes cedex 9

\section{* Correspondance :}

Alexandra Frelau, Oncologie médicale - CLCC Eugène Marquis UNICANCER Avenue de la Bataille Flandres-Dunkerque, 35042 Rennes Cedex, France. Tel.02.99.25.31.96 - a.frelau@rennes.unicancer.fr 


\section{Résumé :}

Les anti-PD-1 s'imposent dernièrement comme un traitement de référence dans de nombreux cancers métastatiques. Ils présentent l'avantage d'une efficacité significative doublée d'un profil de toxicité favorable en monothérapie. Les taux et durées de réponse sont toutefois très variables, ce qui implique, vu leur coût, d'identifier des biomarqueurs prédictifs de réponse. Dans cette revue de la littérature, nous nous intéressons aux biomarqueurs de réponse des immunothérapies anti-PD1 et anti-CTLA-4. L'expression de PD-L1 par les cellules tumorales et du micro-environnement évaluée par immunohistochimie est prédictive de réponse pour certains cancers mais reste une approche mal standardisée avec différents anticorps, différents seuils de positivité et différentes cibles (tumeur ou micro-environnement). II s'agit aussi d'une expression dynamique au cours du temps et hétérogène donc souvent discordante entre biopsies et pièces opératoires. Les lymphocytes circulants pourraient représenter un biomarqueur prédictif notamment via la mesure du ratio polynucléaire neutrophile sur lymphocytes (NLR), ratio simple à réaliser en pratique courante. Un taux de néoantigènes élevé serait également associé à une réponse plus importante ce qui expliquerait les taux de réponses particulièrement élevés des tumeurs avec instabilité micro-satellitaire. Des signatures génomiques pourraient également prédire la sensibilité aux anti-PD1. Par ailleurs, la présence de certaines bactéries de la flore intestinale favoriserait la réponse immunitaire antitumorale même si le mécanisme demeure mal compris. Enfin, des signatures d'expression de cytokines, médiatrices de la réponse immune, pourraient constituer des biomarqueurs pertinents. Plusieurs biomarqueurs de réponse potentiels semblent donc intéressants mais aucun n'est encore prospectivement validé à ce jour.

Mots-clés : Immunothérapie, anti-PD1, biomarqueurs, expression de PD-L1,

\section{Abstract:}

PD-1 checkpoint inhibitors are becoming the reference treatment for several type of cancers. Many patients show remarkable efficacy and low toxicity. However, some patients have a better outcome than others with PD-1 checkpoint inhibitors. So, it is crucial to identify biomarkers of response. We review here the available data of several potential biomarkers of efficacy. The expression of PD-L1, detected by 
immunohistochemistry on tumor cells and immune cells is a good predictive biomarker of response for some cancers; however, this method is not standardized, and there are different antibodies, different cut-off values, and different targets (tumor or microenvironment). Moreover, the expression of PD-L1 is dynamic and heterogeneous within the tumor: expression is discordant between primary tumor and metastasis or between biopsy and surgical specimen. Peripheral blood lymphocytes also can be informative, especially the baseline neutrophil to lymphocyte ratio which is easy to measure in daily practice. High rate of neoantigens is also associated with improved response. Therefore, mutation burden can be predictive of response and this explains why tumors with microsatellites instability have an enhanced response. Similarly, genetic signatures are linked with resistance or response to treatment. Gut microbiota is associated with improved antitumor immune response although the underlying mechanism is not well understood so far. Lastly, it seems that cytokines, mediators of immunity may play a role in the response to immunotherapy and so, constitute an interesting biomarker. Several potential biomarkers are identified but none is prospectively validated so far.

Keywords: PD-1 blockades, immunotherapy, biomarkers, PD-L1 expression 


\section{Introduction}

Plusieurs études récentes ont montré l'intérêt de l'immunothérapie par anti-PD1 dans le traitement de nombreux cancers métastatiques. Les anti-PD1 deviennent ainsi un traitement de choix dans le mélanome métastatique, les cancers bronchopulmonaires, le cancer du rein et les lymphomes d'Hodgkin en rechute ou réfractaires. Les anti-PD1 sont des anticorps monoclonaux se fixant au récepteur PDL1. Les lymphocytes T antitumoraux expriment à leur surface le récepteur PD-1 qui se lie au ligand PD-L1 exprimé par les cellules tumorales ou par les cellules immunosuppressives du micro-environnement tumoral. Cette liaison, habituellement utilisée par l'organisme pour réguler l'homéostasie immunitaire, entraîne l'inactivation des lymphocytes antitumoraux et l'échappement des cellules tumorales à la vigilance de l'immunité cellulaire. Les anti-PD1 vont contrer cette réaction en levant l'inhibition des lymphocytes antitumoraux. Les anti-CTLA-4 fonctionnent sur le même principe mais ciblent la réaction immunologique véhiculée par les cellules présentatrices d'antigènes se déroulant au sein du ganglion lymphatique et non sur le site tumoral. En effet, lors de l'initiation de la réponse immunitaire, au niveau des ganglions lymphatiques, les antigènes sont présentés aux lymphocytes T CD8 par les cellules dendritiques grâce au CMH-I, et aux lymphocytes T CD4 grâce au CMH-II. L'antigène est reconnu par le TCR exprimé par le lymphocyte T. Cette liaison entraîne l'expression de B7 par la cellule dendritique, qui se lie au récepteur CD28 exprimé par le lymphocyte T. Cette liaison permet de stimuler la prolifération lymphocytaire $T$, et la transformation des lymphocytes $T$ naïfs en lymphocytes T actifs. Le récepteur CTLA-4, exprimé par les lymphocytes T, se lie avec une forte affinité au récepteur $B 7$ de la cellule dendritique ce qui entraîne un rétrocontrôle négatif au niveau de l'activation lymphocytaire T. Les anti-CTLA-4 affectent donc la phase d'initiation (phase de " priming ") et d'activation des lymphocytes T. Les anti-PD1 interviennent durant la phase effectrice de l'immunité cellulaire. Le PD-1 est exprimé plus tardivement par les lymphocytes $T$ et se lie aux ligands PD-L1 et PD-L2, exprimés par les cellules tumorales et les cellules présentatrices d'antigènes. Les anti-PD1 ciblent donc la réaction immunitaire ayant lieu dans les tissus périphériques. Ils empêchent la liaison du PD-1 exprimé par le lymphocyte T antitumoral au ligand PD-L1 (ou PD-L2) exprimé par la cellule tumorale et par les cellules immunosuppressives du micro-environnement tumoral $[1,2]$.

Les profils de réponse à ces immunothérapies sont variés, de l'absence de réponse à la réponse complète. Nous avons dans cet article résumé la littérature disponible 
pour différents biomarqueurs potentiels de réponse. Identifier de tels marqueurs pourrait permettre de sélectionner les patients les plus à même de tirer bénéfice de ces traitements et d'en optimiser ainsi les coûts.

\section{L'expression de PD-1/PD-L1}

Le ligand PD-L1 est exprimé à la surface des cellules tumorales et de plusieurs cellules immunitaires présentes dans le micro-environnement tumoral : certains lymphocytes B et $\mathrm{T}$, les macrophages et les cellules dendritiques. Cette expression est en partie induite par I'Interféron gamma (IFN gamma). II est également présent à la surface des cellules vasculaires endothéliales et des cellules des îlots de Langerhans. PD-L2 est l'autre ligand de PD-1, et est présent à la surface des macrophages et des cellules dendritiques, et dans une moindre mesure que PD-L1 à la surface des cellules tumorales. PD-L2, ayant une expression plus restreinte, est impliqué dans l'activation et la polarisation des lymphocytes $T$, tandis que PD-L1 permet de limiter les réactions d'auto-immunité et d'inflammation locale [3-5].

Le pourcentage d'expression de PD-L1 à la surface des cellules est déterminé par immunohistochimie grâce à des anticorps variés. KEYNOTE 001 [6], étude de phase I évaluant le PEMBROLIZUMAB dans le cancer broncho-pulmonaire a utilisé un anticorps de DAKO, le clone 22C3. Elle a montré qu'une expression de PD-L1 d'au moins $50 \%$ des cellules tumorales était prédictive de réponse. Pour les patients atteignant ce seuil, la médiane de survie sans progression (mPFS) était de 6,2 mois contre 3,2 mois chez les patients avec une moindre expression, et la médiane de survie globale (mOS) n'était pas atteinte, contre 12,5 mois. De même, les résultats de CheckMate 067 [7] dans les mélanomes avancés, confrontant les traitements par NIVOLUMAB seul, IPILIMUMAB seul et par l'association IPILIMUMAB et NIVOLUMAB étaient en faveur d'un intérêt prédictif de l'expression de PD-L1. L'anticorps utilisé était le clone 28-8 de DAKO avec un seuil de positivité à $5 \%$ de marquage des cellules tumorales. Pour les patients, dont au moins $5 \%$ des cellules tumorales exprimaient PD-L1, le NIVOLUMAB seul était aussi efficace que l'association NIVOLUMAB et IPILUMUMAB avec une PFS identique à 14 mois. Pour les patients n'atteignant pas ce seuil, le NIVOLUMAB seul était moins efficace avec une PFS de 5,3 mois contre 11,2 mois pour l'association des deux traitements. Contrairement aux deux études précédentes, CheckMate 025 [8], comparant le NIVOLUMAB à I'EVEROLIMUS dans le cancer du rein avancé a montré une amélioration de la survie 
globale avec l'anti-PD1 sans retrouver de différence entre les patients PD-L1 positifs et négatifs. Le seuil retenu était un marquage d' $1 \%$ des cellules tumorales par le clone 28-8. De même CheckMate 032 [9], étude de phase 1-2, considérant I'association NIVOLUMAB et IPILIMUMAB versus NIVOLUMAB seul dans le cancer broncho-pulmonaire à petites cellules, a montré un bénéfice de survie pour la combinaison, indépendant du statut PD-L1, avec des réponses significatives chez des patients PD-L1 négatifs. L'expression de PD-L1 reste assez faible dans ce type de tumeurs, et plus de $75 \%$ des cancers de cette étude avaient un taux d'expression inférieur à $1 \%$.

L'intérêt prédictif de l'expression de PD-L1 n'est donc pas reproductible d'un type tumoral à l'autre. Par ailleurs, différents anticorps sont utilisés avec différents seuils de positivité. Le tableau 1 recense les études de phase III concernant le NIVOLUMAB et le PEMBROLIZUMAB, le tableau 2 des études de phase I-II avec l'ATEZOLIZUMAB et le DURVALUMAB (anti-PD-L1). Ils illustrent le manque de standardisation dans la méthode utilisée : quatre anticorps sont retenus pour l'immunohistochimie (clones 22C3 et 28-8 de DAKO, SP 142 et SP 263 de VENTANA). Les seuils et le type de cellules cibles sont également multiples, de 1, 5, 10 et jusqu'à $50 \%$ des cellules tumorales ou des cellules du micro-environnement.

Une méta-analyse récente [22], rassemblant vingt essais de phases I, II et III a montré que l'expression de PD-L1 était corrélée à la réponse dans le mélanome métastatique où le taux de réponse objective poolée (ORR) était de $45 \%$ pour les patients PD-L1 positifs versus $27 \%$ et dans le carcinome épidermoïde pulmonaire où I'ORR était de $29 \%$ versus $11 \%$. II n'y avait pas, au contraire, de différence significative selon le statut PD-L1 dans le cancer du rein et l'adénocarcinome pulmonaire. Dans tous les cas, dans ces études, la seule expression de PD-L1 ne permettait pas d'identifier de patients sans aucune chance de réponse aux anti-PD1.

Ces résultats sont à nuancer par le fait que nous n'avons pas d'informations claires dans cet article, sur l'origine des échantillons tumoraux : dans les différentes études, l'analyse de PD-L1 a pu être effectuée sur la biopsie initiale ou sur une biopsie après au moins une ligne de traitement systémique (dans CheckMate 067, CheckMate 057 et CheckMate 017, des prélèvements archivés ont pu être utilisés).Or l'expression de PD-L1 est variable au cours du temps. En effet, il s'agit d'une expression dynamique, amenée à se modifier en fonction des différents traitements administrés [23]. Il est 
également démontré que la concentration de PD-L1 varie après traitement du mélanome par anti-PD-L1 car les lymphocytes activés relarguent de I'IFN gamma qui stimule l'expression de PD-L1 à la surface des cellules tumorales environnantes [24]. Certains patients PD-L1 négatifs sont devenus PD-L1 positifs après traitement par anti-PD-L1. De la même manière, une biopsie ne reflète pas l'expression de PD-L1 de toute la tumeur : il existe une hétérogénéité spatiale intra-tumorale mais également inter-tumorale, entre tumeur principale et métastases. Une étude de 2015 [25] a mis en évidence un taux de discordance de $48 \%$ entre l'expression de PD-L1 sur les biopsies et les pièces opératoires ( $74 \%$ de tumeurs n'exprimaient pas PD-L1 contre $26 \%$ des biopsies). Il peut donc paraître inapproprié de se contenter d'une seule biopsie pour évaluer le statut PD-L1 d'un patient. II est possible que les résultats de certaines études, comprenant des échantillons tumoraux réalisés à des moments différents, soient difficiles à interpréter.

Ces données sont toutefois contredites par une étude s'intéressant aux cancers bronchiques, présentée récemment à l'ASCO [26], ne retrouvant pas de différence majeure entre le statut PD-L1 des échantillons récents ou anciens d'un même patient (concordance de 76 \% quand la différence d'âge des échantillons est inférieure à 3 ans), ni de différence d'expression entre la tumeur primaire et la métastase (concordance de 88,6\%). Le clone SP 263 de Ventana a été utilisé pour un cut-off de $25 \%$ d'expression sur les cellules tumorales.

Ainsi, l'expression de PD-L1 pourrait prédire la réponse aux anti-PD1 dans certains types de cancers tels que les cancers pulmonaires et les mélanomes. Cet intérêt prédictif est à nuancer par le manque de standardisation de la méthode utilisant différents anticorps et seuils de positivité, par l'expression probablement hétérogène et dynamique de ce récepteur au cours du temps et au sein même des lésions tumorales.

Concernant la standardisation de la méthode, une étude présentée à l'ASCO [27] en 2016, comparant différents niveaux de positivité de PD-L1 obtenus sur des biopsies de 15 cancers pulmonaires par 4 anticorps différents (clones 28-8, 22C3, SP 142 et SP 263) a rapporté une meilleure homogénéité sur les taux d'expression entre ces anticorps lorsque l'analyse portait sur les cellules tumorales et non sur celles du micro-environnement. Les résultats obtenus avec les différents anticorps ne restaient cependant pas interchangeables. D'autres études seront nécessaires pour valider prospectivement ces résultats, ainsi que pour déterminer le seuil de 
positivité pour chaque anticorps. Une étude plus récente [28] a décrit une importante corrélation entre les résultats obtenus par ces 4 clones dans le cancer broncho-pulmonaire. Mais le clone SP263 identifiait plus de cas positifs sur l'analyse des cellules du micro-environnement que les autres clones. Des tests supplémentaires seront nécessaires pour mieux comprendre ces différences de résultats entre les anticorps.

Une autre méthode, alternative à l'immunohistochimie pourrait également se développer pour déterminer le seuil d'expression de PD-L1: une étude [29] a utilisé I'ATEZOLIZUMAB marqué au Cuivre-64 ( $\left[{ }^{64} \mathrm{Cu}\right]$ atezolizumab) chez des souris porteuses d'une tumeur exprimant PD-L1. Des images ont ensuite été réalisées au TEP-Scanner, mettant en évidence que la distribution du traceur était corrélée à l'expression de PD-L1. Cette méthode pourrait permettre d'évaluer l'expression de PD-L1 de manière standardisée, et de mieux la suivre dans le temps et l'espace, sans avoir recours à de multiples biopsies. II sera nécessaire de confirmer l'intérêt de cette méthode par des études ultérieures, notamment sur l'homme.

Si l'expression de PD-L1peut être corrélée à la réponse aux anti-PD1, l'existence de patients répondeurs chez les patients n'exprimant pas PD-L1 (valeur prédictive négative insuffisante) limite à ce jour l'impact de ce marqueur dans la décision thérapeutique.

\section{Le taux de lymphocytes et leucocytes}

Plusieurs études [30,31] ont montré que des taux élevés de certains types de lymphocytes étaient associés à une meilleure réponse. Un fort taux de lymphocytes T CD8+ PD1+ sanguins avant traitement serait associé à une meilleure réponse à I'immunothérapie. Une diminution des lymphocytes T PD1+ intra-tumoraux après anti-PD1 serait quant à elle prédictive de réponse au traitement [31]. La détection de lymphocytes TCD8+ dans l'environnement tumoral, cellule effectrice de l'immunité antitumorale, est centrale dans la réponse aux inhibiteurs de checkpoint. La présence d'infiltrats lymphocytaires péri-tumoraux avant traitement (TILS = Tumor Infiltrated Lymphocytes) est liée à une augmentation de la réponse aux anti-PD1 $[32,33]$. Une étude [34] a mis en évidence une augmentation de l'infiltration tumorale par les lymphocytes T après 2 cures de NIVOLUMAB chez la majorité des patients (cancer du rein métastatique). Une prolifération des lymphocytes $T$ 
CD8+intra-tumoraux pendant le traitement par anti-PD1 serait directement corrélée à l'efficacité antitumorale [33]. Ces différents résultats ont été confirmés par une étude plus récente dans le mélanome métastatique sous anti-PD1 montrant que les taux de lymphocytes T PD1+ intra-tumoraux et péri-tumoraux étaient 5 à 7 fois plus importants avant traitement chez les patients répondeurs que chez les non répondeurs et que le taux de lymphocytes $T C D 8+$ augmentait de manière significative chez les répondeurs après 2 mois de traitement [35].

Dans le mélanome métastatique sous IPILIMUMAB, la présence de lymphocytes T CD8+ Ki67+ serait de pronostic favorable. La protéine Ki67 est un marqueur cellulaire de prolifération et de division cellulaire. Les lymphocytes T Ki67+ sont donc des lymphocytes en prolifération [36].

Un type particulier [37] de lymphocytes T CD4 ICOS+ (lymphocytes mémoires activés), pourrait également être associé à une meilleure réponse tumorale. Sous anti-CTLA-4, la liaison B7-CD28 permet l'activation des lymphocytes T avec expression d'ICOS. Dans une étude sur 27 patients traités par IPILIMUMAB pour mélanome métastatique, un fort taux de lymphocytes T CD4 ICOS+ circulants était associé à une meilleure réponse. Une augmentation de ce taux après 7 semaines de traitement serait prédictive de taux de réponse et de survie globale majorés. Un fort taux de lymphocytes $T C D 3+C D 4+I C O S+C D 45 R O+$ était plus particulièrement associé à une augmentation de la survie dans le mélanome métastatique sous IPILIMUMAB [38]. ICOS pourrait également être utilisé comme un marqueur pharmacodynamique de l'efficacité biologique de l'IPILIMUMAB : une augmentation significative du taux de lymphocytes T CD4+ ICOS + a été détectée chez des patients avec un mélanome métastatique ou un cancer de la vessie [39]. ICOS serait donc le témoin de l'activité biologique de I'IPILUMUMAB qui vise à restaurer l'activation des lymphocytes Tà la phase précoce de l'immunité cellulaire.

Chez des patients atteints de carcinome urothélial traités par ATEZOLIZUMAB, un haut niveau d'infiltration lymphocytaire T péri-tumoral, ainsi qu'une forte clonalité intra-tumorale puis une expansion sanguine des clones dominants étaient associés à une meilleure survie [40]. L'ATEZOLIZUMAB permettrait donc la sélection de quelques clones lymphocytaires spécifiques ensuite retrouvés dans le sang périphérique. Une faible variabilité des lymphocytes circulants après traitement était associée positivement à la survie. Une autre étude a confirmé ces résultats [41]. Un répertoire varié de TCR avant traitement dans le sang périphérique favoriserait la 
probabilité d'avoir un ou plusieurs clones antitumoraux. L'augmentation de clones lymphocytaires spécifiques dans le sang périphérique après traitement montrerait que les anti-PD-L1 ont permis la sélection de lymphocytes T péri tumoraux spécifiques ensuite retrouvés dans le sang circulant. II est prouvé que la liaison PD1PD-L1 exerce un rétrocontrôle négatif sur l'expression du TCR par les lymphocytes T [42]. Cela entraîne une diminution de la prolifération et des activités cytotoxiques des lymphocytes T [43]. L'inhibition de cette interaction (par l'ATEZOLIZUMAB) pourrait donc expliquer l'augmentation de lymphocytes T antitumoraux, dont le TCR reconnaît les antigènes tumoraux, passant ensuite dans le sang périphérique. De même, une forte diversité du TCR avant traitement par IPILIMUMAB dans le mélanome métastatique serait associée à un meilleur bénéfice clinique [44]. Une diversification du répertoire TCR a été constatée après anti-CTLA-4 [45]. II n'y aurait pas d'expansion d'un clone dominant mais de plusieurs clones différents. Les antiCTLA-4 agissant sur la phase d'activation des lymphocytes ayant lieu dans le ganglion, il y aurait une activation de différents clones lymphocytaires.

Une autre étude [46] concernant des patients porteurs de cancer de la prostate sous IPILIMUMAB a montré de même qu'une augmentation du répertoire de lymphocytes T après le début du traitement était liée à une augmentation de la réponse clinique mais également de la toxicité.

Le ratio neutrophiles/lymphocytes [47] (NLR) pourrait lui aussi constituer un biomarqueur pronostique voire prédictif. Une étude rétrospective sur 187 patients, traités par IPILIMUMAB pour mélanome métastatique a montré ainsi qu'un NLR inférieur à cinq était associé à une augmentation de la survie globale (mOS de 8 mois versus 2,6 ) et de la survie sans progression (mPFS de 3,6 mois versus 1,9). Ce ratio, facile à utiliser en pratique, pourrait permettre d'appréhender précocement les patients répondeurs au traitement : les patients ayant une augmentation du ratio NLR en début de traitement sembleraient ainsi avoir une survie sans progression plus courte [48]. Ces résultats ont été confirmés dans une étude plus récente [49] montrant qu'un taux de lymphocytes supérieur à $1000 / \mathrm{mm} 3$ et un taux de neutrophiles inférieurs à $4000 / \mathrm{mm} 3$ étaient associés à une meilleure OS, après la $1^{\text {re }}$ et la $2^{\mathrm{e}}$ injection de NIVOLUMAB (mélanome non résécable). Les taux avant traitement n'étaient pas prédictifs de réponse.

Une étude rétrospective [50] concernant 73 patients traités par IPILIMUMAB pour mélanome métastatique a montré qu'un taux de lymphocytes supérieur à 1000 par 
$\mathrm{mm} 3$ après la première injection de traitement était associé à une augmentation de la mOS (11,1 mois versus 4,8 mois). De même, le taux d'éosinophiles a été étudié. Une augmentation de ce taux de plus de $100 \mathrm{par} \mathrm{mm}^{3}$ entre la première et la deuxième injection était liée à une augmentation de la mOS (11,3 mois versus 6,8 mois). Ces résultats sont concordants avec ceux d'une étude récente [51] montrant une augmentation du taux de réponse ( $35 \%$ versus $15 \%$ ) chez les patients ayant une augmentation du taux d'éosinophiles de plus de $100 / \mathrm{mm}^{3}$ entre la $2 \mathrm{e}$ et la $4 \mathrm{e}$ injection.

Les taux intra tumoraux ou circulants de certains sous types leucocytaires pourraient donc précocement apprécier la réponse, en particulier le ratio neutrophile sur lymphocytes qui est simple et peu coûteux à réaliser en routine. II manque cependant aussi pour ces paramètres des données prospectives de validation définitive.

\section{Charge mutationnelle et taux de néo antigènes}

Les mutations de l'ADN des cellules tumorales génèrent des peptides oncogéniques à l'origine d'antigènes de surface, reconnus comme étrangers au soi par le système immunitaire, appelés néo antigènes. Ces néo antigènes sont eux-mêmes à l'origine de l'activation et de la prolifération de lymphocytes T antitumoraux. Les anti-PD1 et les anti-CTLA-4 visant à empêcher l'inactivation de ces lymphocytes cytotoxiques, le taux de néo antigènes pourrait être prédictif de la réponse à ces immunothérapies. Une étude concernant 45 patients porteurs d'un cancer métastatique avec ou sans défaut du système de réparation des mésappariements de I'ADN (MMR = MisMatchRepair) [52] a retrouvé un taux de réponse de $40 \%$ chez les patients avec MMR déficient contre un taux de réponse de $0 \%$ chez ceux dont le système de réparation de l'ADN n'était pas altéré. Cette déficience du système MMR est à I'origine de très nombreuses mutations donc d'un grand nombre de néo antigènes. II concerne $15 \%$ des cancers colorectaux sporadiques mais $100 \%$ des patients porteurs de syndromes de Lynch.

Une étude parue dans le NEJM en 2014 s'est intéressée à I'IPILIMUMAB dans les mélanomes métastatiques et a déterminé une " signature antigénique " associée à une meilleure réponse dans une cohorte-test. Cette signature a été validée dans une cohorte de validation de 39 patients avec une différence de survie globale en faveur 
des patients présentant cette signature [53]. Celle-ci contenait entre autres des antigènes semblables à ceux des virus et bactéries commensaux dont les antigènes sont donc fréquemment pris en charge par les lymphocytes.

Une seconde étude concernant la charge mutationnelle, parue dans Science en 2015, a établi un seuil à 178 mutations au-delà duquel un bénéfice durable du PEMBROLIZUMAB était observé parmi les patients porteurs d'un cancer bronchopulmonaire. Ce seuil a été validé dans un groupe contrôle (mPFS de 14,5 mois contre 3,5 mois) [54]. Parmi ces mutations, se trouvaient celles de gènes impliqués dans la réplication et la réparation de l'ADN, comme POLD1, POLE, MSH2. Un fort taux de transversions (remplacement d'une purine par une pyrimidine ou inversement), correspondant à la signature moléculaire des patients fumeurs a également été retrouvé et était l'origine d'un plus grand nombre de néo antigènes.

Une association entre charge mutationnelle et infiltration lymphocytaire a été démontrée [55]: un fort taux de mutations serait lié à une augmentation des lymphocytes T CD4 et CD8+ activés péri-tumoraux. Une telle association a également été trouvée entre défaut de réparation de l'ADN et charge mutationnelle totale. Cela dit, seules les tumeurs avec défaut de réparation des mésappariements de l'ADN présentaient une augmentation de l'infiltration lymphocytaire ce qui plaide aussi pour faire du statut MMR un biomarqueur prédictif de réponse.

Le taux de néo antigènes et la charge mutationnelle semblent donc intéressants pour prédire la réponse mais les méthodes de séquençage du génome s'avèrent à ce jour encore complexes et coûteuses à réaliser en pratique. Par ailleurs, les tumeurs avec instabilité micro-satellitaire, les plus à même de répondre, concernent une faible proportion de patients.

\section{Signatures d'expression génique et mutations}

Certaines études via des méthodes de séquençage du génome, se sont intéressées à l'impact prédictif de signatures géniques.

Une étude a décrit ainsi un type de tumeurs présentant une réponse immune innée liée aux dommages à I'ADN [56]. Il existe dans ces tumeurs une surproduction de cytokines augmentant l'infiltration lymphocytaire et une surexpression de PD-L1. Une signature de 44 gènes, constituant un phénotype DDRD (DNA Damage Response 
Deficient), a été identifiée. Cette signature, constituant un test, a été appliquée à un ensemble de cancers colorectaux. Les résultats ont montré que $80 \%$ des tumeurs MSI (microsatellites instables) et $25 \%$ des tumeurs MSS (microsatellites stables) présentaient cette signature (et ont donc un test positif). Ce test détecterait de fait les patients porteurs de tumeurs déficientes pour la réparation de l'ADN et permettrait d'identifier les patients répondeurs aux anti-PD-1. Cette signature pourrait de surcroît permettre d'identifier les rares patients MSS répondeurs.

Une autre étude a évalué des signatures contenant des gènes liés à la réponse immune et associés à un meilleur taux de réponse aux anti-PD-1 dans différents types de cancers [57]. Parmi ces signatures, 15 étaient constituées par des gènes codant pour la réponse des lymphocytes T cytotoxiques. Ces résultats ont été appuyés par une étude montrant un lien entre stabilité tumorale et survie sans progression avec une signature de 6 gènes codant en majeure partie pour l'activation d'effecteurs cellulaires du système immunitaire (Lymphocytes T, NK, T régulateurs...) [58].

Un article récent [59] a montré un lien entre charge mutationnelle élevée et amélioration de la survie globale et, dans une moindre mesure, de la réponse à l'immunothérapie. II existait parmi les patients répondeurs et non répondeurs des patients avec une faible et forte charge mutationnelle. Certains patients répondeurs avec un plus faible taux de mutations avaient une meilleure survie que les patients non répondeurs avec un fort taux de mutations, ce qui laisserait supposer que des gènes spécifiques influencent la réponse. Une mutation du gène BRCA2 a été ainsi fréquemment retrouvée parmi les patients répondeurs. II a également été mis en évidence une signature transcriptionnelle spécifique de résistance innée aux antiPD-1 (IPRES pour Innate anti-PD-1 Resistance). Cette signature montrait une surexpression de certains gènes parmi lesquels des gènes impliqués dans la transition épithélio-mésenchymateuse, l'angiogenèse, le remodelage de la matrice extra-cellulaire, l'adhésion cellulaire ou la cicatrisation (AXL, ROR2, WTN5A, LOXL2,...). Certains gènes pourraient donc influencer favorablement ou défavorablement la réponse à l'immunothérapie. Dans une étude [60] concernant des mélanomes traités par anti-CTLA-4, les mutations de deux gènes, SERPINB3 et SERPINB4 étaient liées à la réponse aux anti-CTLA-4 et à la survie globale. Ces deux gènes codent pour des inhibiteurs de protéase ayant un rôle dans l'apoptose et dans l'auto-immunité. Leur mutation n'avait cependant pas d'influence sur la survie des patients qui n'étaient pas traités par immunothérapie. À l'inverse, un autre travail 
récent a montré l'impact prédictif de non-réponse de certaines mutations du gène de la beta-2-microglobuline et des gènes JAK1 et 2, respectivement impliqués dans l'expression du complexe majeur d'histocompatibilité de classe I et dans la réponse à I'interféron gamma [61].

Ainsi certaines mutations et signatures génétiques spécifiques pourraient s'avérer utiles pour distinguer les répondeurs des non-répondeurs. Ces résultats restent, pour le moment, à valider sur de plus grandes cohortes mais auront probablement un rôle majeur dans la personnalisation de l'immunothérapie.

\section{Le microbiote}

La flore intestinale rassemble plus de 100000 milliards de bactéries vivant en accord avec leur hôte, en favorisant le métabolisme et le rendement énergétique alimentaire et en limitant l'invasion par des agents pathogènes. D'une manière encore partiellement incomprise, l'exposition des lymphocytes à certains types de bactéries de la flore commensale peut faire varier les réponses à l'immunothérapie antitumorale [62]. Une étude parue dans Science [63] a analysé la croissance de mélanomes sur un modèle murin comprenant deux types de souris : les souris JAX à fort taux de lymphocytes intra-tumoraux d'un côté et souris TAC à plus faible taux de I'autre. La croissance tumorale était beaucoup plus faible chez les souris JAX, du fait de l'infiltration de la tumeur par les lymphocytes intra-tumoraux. L'immunothérapie par anti-PDL-1 était également plus efficace chez les souris JAX. Le mélange de souris ou la greffe de matières fécales de souris JAX conférait une résistance aux souris TAC, suggérant un lien transmissible lié à la flore commensale. Une bactérie spécifique a pu être identifiée : Bifidobacterium. Cette bactérie particulière confère à son hôte une résistance innée aux tumeurs ainsi qu'une meilleure réponse aux immunothérapies. Une deuxième étude parue dans Science [64] concernant toujours un modèle murin porteur de mélanomes traités par anti-CTLA-4, a montré que les souris répondaient moins bien à l'immunothérapie après destruction de leur flore intestinale par une antibiothérapie à large spectre.

Il existe donc un lien étroit entre réaction immunitaire antitumorale et microbiote intestinal et donc un lien entre réponse à l'immunothérapie et ce même microbiote. Le mécanisme reste pour le moment mal compris mais son élucidation pourrait permettre de confirmer la valeur de ce potentiel biomarqueur prédictif. II est 
possible que l'existence de mimétismes moléculaires entre les motifs antigéniques présents sur les bactéries commensales et les néo antigènes tumoraux puisse expliquer une meilleure réponse immunitaire antitumorale selon l'exposition à certaines bactéries [64]. Les antigènes présents à la surface des bactéries intestinales pourraient prédisposer le système immunitaire à reconnaître certains néo antigènes tumoraux similaires [65-67]. Lors de la réponse immunitaire antitumorale dirigée contre les néo antigènes tumoraux, il pourrait exister une réactivité croisée avec les antigènes bactériens ou viraux auxquels l'organisme a été exposé [53].

La clarification de ces interactions pourrait même permettre de modifier la flore commensale d'un individu pour améliorer sa réponse immunitaire antitumorale et donc de favoriser sa réponse à l'immunothérapie.

\section{Les cytokines}

Les cytokines, groupe de protéines ayant un rôle de médiateur entre les différentes cellules immunitaires, sont notamment produites par les cellules immunitaires, et permettent la régulation des processus immuns en se fixant sur leurs récepteurs spécifiques.

Il y a des cytokines activatrices comme l'IFN-gamma, les TNF (Tumor Necrosis Factor)-alpha et béta produites par les T cytotoxiques. Ces 3 cytokines contribuent à la défense antitumorale par différents mécanismes. L'IFN-gamma, en association aux TNF, a des propriétés cytotoxiques directes, inhibe la croissance tumorale, active les macrophages, améliore l'expression des molécules du $\mathrm{CMH}$, favorisant ainsi la reconnaissance de l'antigène. Parmi les cytokines inhibitrices, le rôle du TGF (Transforming Growth Factor)-béta est à souligner. C'est une cytokine polypeptidique, produite de manière ubiquitaire dans l'organisme. Elle a un rôle complexe, avec une fonction immunosuppressive sur de nombreuses cellules de I'immunité innée ou adaptative et est notamment produite par les lymphocytes T CD4 régulateurs visant à modérer la réaction immune. Elle peut également prévenir la carcinogénèse. Cette cytokine permet le maintien de l'homéostasie tissulaire, régule la prolifération, la différenciation, l'adhésion et module le microenvironnement cellulaire. Les cellules tumorales peuvent inactiver le récepteur du TGF-béta ou altérer les fonctions anti-tumorales de cette voie. Dans ce dernier cas, la 
tumeur acquiert un fort potentiel de division et de progression [68].

Les cytokines ont un rôle clé dans l'immunité antitumorale et pourraient donc constituer un bio marqueur intéressant de réponse à l'immunothérapie.

Une étude [69] dernièrement présenté à l'ASCO, constatant que les lymphocytes activés relarguaient certaines cytokines (IFN-gamma, IL2-4-5-10, TNF-alpha et béta) favorisant l'afflux de cellules immunitaires sur le site tumoral et augmentant la reconnaissance des cellules tumorales par les cellules cytotoxiques, a décrit un test permettant de mesurer les taux de cytokines avec une bonne sensibilité et une bonne spécificité. Ce test pourrait donc servir d'outil préférentiel pour la mesure de cytokines prédictives de réponse. Un essai de phase II[70], concernant le mélanome avancé sous IPILIMUMAB, a affirmé l'importance du micro-environnement tumoral dans la réponse à l'immunothérapie et a souligné le rôle de I'IDO (indol-amine 2,3dioxygenase) dont l'expression était associée positivement au bénéfice clinique de I'IPILIMUMAB . L'IDO est une enzyme dégradant le tryptophane dans l'environnement tumoral et est exprimée par les cellules tumorales, dans les ganglions drainants, par le placenta, par plusieurs cellules du système immunitaire notamment les macrophages, les cellules dendritiques et les cellules myéloïdes suppressives (MDSC) [71]. Elle entraîne une suppression des fonctions effectrices des lymphocytes T, bloque leur différenciation et induit leur apoptose [72]. Elle a donc une fonction immunosuppressive. Mais l'expression de cette enzyme est en particulier provoquée par la sécrétion de cytokines pro-inflammatoires telles que I'IL-1, I'IFN-gamma et le TNF- alpha qui témoignent d'une forte activation du système immunitaire. Il serait donc possible que I'IDO soit surexprimée chez les patients ayant une forte réponse immunitaire antitumorale, d'où une augmentation d'efficacité de l'immunothérapie chez ces patients [70]. Le dosage de certaines cytokines au cours des thérapies par anti-PD-1 pourrait ainsi être informatif quant à la réponse au traitement. Une autre étude [73], s'est intéressée au rôle du TGF-béta. Le TGF-béta est fréquemment surexprimé dans les cancers agressifs, corrélé à une augmentation des métastases, à un pronostic défavorable et à une résistance aux différents traitements. Certaines études précliniques ont montré que les antagonistes du TGF-béta permettaient d'augmenter l'efficacité des chimiothérapies et des immunothérapies. Mais le TGF-béta aurait également un rôle dans l'acquisition du phénotype EMT (Epithelial Mesenchymal Transition), permettant aux cellules tumorales d'acquérir un potentiel plus invasif. Or ce phénotype, caractérisé par une signature génétique particulière dans le cancer du poumon non à petites 
cellules, serait associé à une surexpression de PD-L1. Cette catégorie de patients, ayant le phénotype EMT, pourrait donc bénéficier au mieux des anti-PD-1. Une étude récente [74] a mis en évidence une variation dans l'expression de PD-L1 au cours de la transition épithélio-mésenchymateuse dans le cancer bronchopulmonaire non à petites cellules. Le phénotype EMT a été induit avec un traitement par TGF-béta ou chimiothérapie. Une surexpression de PD-L1 a été constatée après induction par le TGF-béta. La chimiothérapie provoquait également une augmentation de la sécrétion de TGF-béta à l'origine d'une surexpression de PD-L1. L'expression de PD-L1 serait donc en partie régulée par le TGF-béta. Une association significative entre l'expression de PD-L1 et le phénotype EMT a également été retrouvée. Le TGF-béta aurait donc un rôle potentiellement modulateur de la réponse aux anti-PD1, mais son rôle dans la réaction immune étant complexe, il semble également difficile pour le moment de le considérer comme un biomarqueur de réponse.

Ainsi les cytokines, intervenant dans l'activation ou l'inhibition de la réponse immunitaire antitumorale, ont probablement un impact dans la réponse aux immunothérapies, mais leurs actions sont complexes et des études complémentaires sont nécessaires pour déterminer lesquelles d'entre elles seraient les plus à même de prédire la réponse aux inhibiteurs de checkpoint immunitaires.

\section{Conclusion}

Ces dernières années ont vu l'essor des immunothérapies anti-CTLA-4 et surtout, anti-PD-1. Ces thérapies permettent d'obtenir des réponses objectives durables mais seulement chez 20 à $30 \%$ des patients. Identifier un biomarqueur de réponse fiable pourrait permettre de n'administrer ces traitements qu'aux seuls patients susceptibles d'en bénéficier durablement avec un intérêt en termes de stratégie thérapeutique mais également de coût de santé étant donné le prix élevé de ces traitements. II n'existe pas à ce jour de biomarqueur parfaitement discriminant. Nous avons considéré dans cet article six biomarqueurs potentiels, illustrés par le schéma ci-dessous (figure 1).L'expression de PD-L1 en IHC pourrait être pertinente dans certaines conditions mais il sera pour cela indispensable de définir une méthode d'immunohistochimie standard et de fixer un seuil de positivité consensuel. 
Le taux de lymphocytes, et plus particulièrement le ratio polynucléaires neutrophiles sur lymphocytes pourrait permettre d'apprécier de manière précoce la réponse mais ce marqueur nécessite une validation prospective.

La détermination du taux de néo antigènes est à ce jour difficile à réaliser en routine mais ce paramètre est potentiellement très pertinent à visée prédictive, comme l'illustre le cas des tumeurs avec déficience du système MMR. Certaines signatures géniques spécifiques, et plus particulièrement la présence de certaines mutations auraient également un impact majeur dans la réponse thérapeutique et pourraient permettre une personnalisation de l'immunothérapie des cancers.

Le microbiote a une influence dans la réponse à l'immunothérapie qui reste mal comprise à ce jour et qui doit donc être approfondie avant de pouvoir générer un biomarqueur voire même de constituer une approche thérapeutique synergique aux anti-PD-1.

Enfin, certaines cytokines, agents essentiels au bon déroulement de la réaction immunitaire, favorisant ou inhibant la réponse anti tumorale, pourraient théoriquement servir de biomarqueurs mais des études complémentaires seraient nécessaires pour les identifier et valider leur valeur prédictive. Leur action sur la réaction immune est par ailleurs complexe et donc leur rôle prédictif difficile à apprécier...

La question d'un biomarqueur de réponse aux anti-PD-1 (et aux anti-CTLA-4) n'est donc pas résolue et reste un enjeu majeur afin de pouvoir personnaliser et optimiser le coût de ces traitements.

\section{Remerciements:}

Les auteurs souhaitent en particulier remercier Odile Audrain, documentaliste au CLCC Eugène Marquis pour l'aide apportée à la rédaction de cet article et pour la gestion de la bibliographie.

\section{Déclaration de liens d'intérêts :}




\section{Références}

[1] Sharma P, Wagner K, Wolchok JD, Allison JP. Novel cancer immunotherapy agents with survival benefit: recent successes and next steps. Nat Rev Cancer 2011;11:805-12.

[2] Buchbinder EI, Desai A. CTLA-4 and PD-1 Pathways: Similarities, Differences, and Implications of Their Inhibition. Am J Clin Oncol 2016;39:98-106.

[3] Guan J, Lim KS, Mekhail T, Chang C-C. Programmed Death Ligand-1 (PD-L1) Expression in the Programmed Death Receptor-1 (PD-1)/PD-L1 Blockade: A Key Player Against Various Cancers. Arch Pathol Lab Med 2017;141:851-61.

[4] He J, Hu Y, Hu M, Li B. Development of PD-1/PD-L1 Pathway in Tumor Immune Microenvironment and Treatment for Non-Small Cell Lung Cancer. Sci Rep 2015;5:13110.

[5] Chen DS, Irving BA, Hodi FS. Molecular pathways: next-generation immunotherapy--inhibiting programmed death-ligand 1 and programmed death-1. Clin Cancer Res Off J Am Assoc Cancer Res 2012;18:6580-7.

[6] Garon EB, Rizvi NA, Hui R, Leighl N, Balmanoukian AS, Eder JP, et al. Pembrolizumab for the treatment of non-small-cell lung cancer. N Engl J Med 2015;372:2018-28.

[7] Larkin J, Chiarion-Sileni V, Gonzalez R, Grob JJ, Cowey CL, Lao CD, et al. Combined Nivolumab and Ipilimumab or Monotherapy in Untreated Melanoma. N Engl J Med 2015;373:23-34.

[8] Motzer RJ, Escudier B, McDermott DF, George S, Hammers HJ, Srinivas S, et al. Nivolumab versus Everolimus in Advanced Renal-Cell Carcinoma. N Engl J Med 2015;373:1803-13.

[9] Antonia SJ, López-Martin JA, Bendell J, Ott PA, Taylor M, Eder JP, et al. Nivolumab alone and nivolumab plus ipilimumab in recurrent small-cell lung cancer (CheckMate 032): a multicentre, open-label, phase 1/2 trial. Lancet Oncol 2016;17:883-95.

[10] Weber JS, D'Angelo SP, Minor D, Hodi FS, Gutzmer R, Neyns B, et al. Nivolumab versus chemotherapy in patients with advanced melanoma who progressed after anti-CTLA-4 treatment (CheckMate 037): a randomised, controlled, open-label, phase 3 trial. Lancet Oncol 2015;16:375-84.

[11] Larkin J, Chiarion-Sileni V, Gonzalez R, Grob JJ, Cowey CL, Lao CD, et al. Combined Nivolumab and Ipilimumab or Monotherapy in Untreated Melanoma. N Engl J Med 2015;373:23-34.

[12] Borghaei H, Paz-Ares L, Horn L, Spigel DR, Steins M, Ready NE, et al. Nivolumab versus Docetaxel in Advanced Nonsquamous Non-Small-Cell Lung Cancer. N Engl J Med 2015;373:1627-39.

[13] Robert C, Long GV, Brady B, Dutriaux C, Maio M, Mortier L, et al. Nivolumab in previously untreated melanoma without BRAF mutation. N Engl J Med 2015;372:320-30.

[14] Brahmer J, Reckamp KL, Baas P, Crinò L, Eberhardt WEE, Poddubskaya E, et al. Nivolumab versus Docetaxel in Advanced Squamous-Cell Non-Small-Cell Lung Cancer. N Engl J Med 2015;373:123-35. 
[15] Antonia SJ, López-Martin JA, Bendell J, Ott PA, Taylor M, Eder JP, et al. Nivolumab alone and nivolumab plus ipilimumab in recurrent small-cell lung cancer (CheckMate 032): a multicentre, open-label, phase 1/2 trial. Lancet Oncol 2016;17:883-95.

[16] Robert C, Schachter J, Long GV, Arance A, Grob JJ, Mortier L, et al. Pembrolizumab versus Ipilimumab in Advanced Melanoma. N Engl J Med 2015;372:2521-32.

[17] Herbst RS, Baas P, Kim D-W, Felip E, Pérez-Gracia JL, Han J-Y, et al. Pembrolizumab versus docetaxel for previously treated, PD-L1-positive, advanced non-small-cell lung cancer (KEYNOTE-010): a randomised controlled trial. Lancet Lond Engl 2016;387:1540-50.

[18] Pembrolizumab (MK-3475) Versus Standard Treatment for Recurrent or Metastatic Head and Neck Cancer (MK-3475-040/KEYNOTE-040) - Full Text View - ClinicalTrials.gov n.d. https://clinicaltrials.gov/ct2/show/NCT02252042 (accessed April 24, 2017).

[19] Reck M, Rodríguez-Abreu D, Robinson AG, Hui R, Csőszi T, Fülöp A, et al. Pembrolizumab versus Chemotherapy for PD-L1-Positive Non-Small-Cell Lung Cancer. N Engl J Med 2016;375:1823-33.

[20] Fehrenbacher L, Spira A, Ballinger M, Kowanetz M, Vansteenkiste J, Mazieres J, et al. Atezolizumab versus docetaxel for patients with previously treated non-small-cell lung cancer (POPLAR): a multicentre, open-label, phase 2 randomised controlled trial. Lancet Lond Engl 2016;387:1837-46.

[21] Hoffman-Censits JH, Grivas P, Van Der Heijden MS, Dreicer R, Loriot Y, Retz M, et al. IMvigor 210, a phase II trial of atezolizumab (MPDL3280A) in platinum-treated locally advanced or metastatic urothelial carcinoma (mUC). J Clin Oncol 2016;34:355-355.

[22] Gandini S, Massi D, Mandalà M. PD-L1 expression in cancer patients receiving anti PD-1/PD-L1 antibodies: A systematic review and meta-analysis. Crit Rev Oncol Hematol 2016;100:88-98.

[23] Sharma P, Allison JP. The future of immune checkpoint therapy. Science 2015;348:56-61.

[24] Powderly JD, Koeppen H, Hodi FS, Sosman JA, Gettinger SN, Desai R, et al. Biomarkers and associations with the clinical activity of PD-L1 blockade in a MPDL3280A study [abstract]. J Clin Oncol 2013;31:3001-3001.

[25] Ilie M, Long-Mira E, Bence C, Butori C, Lassalle S, Bouhlel L, et al. Comparative study of the PD-L1 status between surgically resected specimens and matched biopsies of NSCLC patients reveal major discordances: a potential issue for anti-PD-L1 therapeutic strategies. Ann Oncol Off J Eur Soc Med Oncol 2016;27:147-53.

[26] Midha A, Sharpe A, Scott M, Walker J, Shi K, Ballas M, et al. PD-L1 expression in advanced NSCLC: Primary lesions versus metastatic sites and impact of sample age [abstract]. J Clin Oncol 2016;34(15):3025.

[27] Scheel AH, Dietel M, Heukamp LC, Jöhrens K, Kirchner T, Reu S, et al. Diagnostic PD-L1 immunohistochemistry in NSCLC: Results of the first German harmonization study [abstract]. J Clin Oncol 2016;34.

[28] Parra ER, Villalobos P, Mino B, Rodriguez-Canales J. Comparison of Different Antibody Clones for Immunohistochemistry Detection of Programmed Cell Death Ligand 1 (PD-L1) on Non-Small Cell Lung Carcinoma. Appl Immunohistochem Mol Morphol 2017 Jul 17

[29] Lesniak WG, Chatterjee S, Gabrielson M, Lisok A, Wharram B, Pomper MG, et al. PD-L1 Detection in Tumors Using [(64)Cu]Atezolizumab with PET. Bioconjug Chem 2016;27:2103-10. 
[30] Dronca RS. Frequency of certain immune cells in blood may predict metastatic melanoma response to Pembrolizumab, New York: CRI-CIMT-EATI-AACR International Cancer Immunotherapy Conference; Sept.

[31] Ngiow SF, Young A, Jacquelot N, Yamazaki T, Enot D, Zitvogel L, et al. A Threshold Level of Intratumor CD8+ T-cell PD1 Expression Dictates Therapeutic Response to Anti-PD1. Cancer Res 2015;75:3800-11.

[32] Teng MWL, Ngiow SF, Ribas A, Smyth MJ. Classifying Cancers Based on T-cell Infiltration and PD-L1. Cancer Res 2015;75:2139-45.

[33] Tumeh PC, Harview CL, Yearley JH, Shintaku IP, Taylor EJM, Robert L, et al. PD-1 blockade induces responses by inhibiting adaptive immune resistance. Nature 2014;515:568-71.

[34] Choueiri TK, Fishman MN, Escudier B, McDermott DF, Drake CG, Kluger H, et al. Immunomodulatory Activity of Nivolumab in Metastatic Renal Cell Carcinoma. Clin Cancer Res Off J Am Assoc Cancer Res 2016;22:5461-71.

[35] Vilain RE, Menzies AM, Wilmott JS, Kakavand H, Madore J, Guminski A, et al. Dynamic Changes in PD-L1 Expression and Immune Infiltrates Early During Treatment Predict Response to PD-1 Blockade in Melanoma. Clin Cancer Res Off J Am Assoc Cancer Res 2017;23:5024-33.

[36] Wang W, Yu D, Sarnaik AA, Yu B, Hall M, Morelli D, et al. Biomarkers on melanoma patient T cells associated with ipilimumab treatment. J TransI Med 2012;10:146.

[37] Di Giacomo AM, Calabrò L, Danielli R, Fonsatti E, Bertocci E, Pesce I, et al. Long-term survival and immunological parameters in metastatic melanoma patients who responded to ipilimumab $10 \mathrm{mg} / \mathrm{kg}$ within an expanded access programme. Cancer Immunol Immunother CII 2013;62:1021-8.

[38] Di Giacomo AM, Ascierto PA, Queirolo P, Pilla L, Ridolfi R, Santinami M, et al. Three-year follow-up of advanced melanoma patients who received ipilimumab plus fotemustine in the Italian Network for Tumor Biotherapy (NIBIT)-M1 phase II study. Ann Oncol Off J Eur Soc Med Oncol 2015;26:798-803.

[39] Ng Tang D, Shen Y, Sun J, Wen S, Wolchok JD, Yuan J, et al. Increased frequency of ICOS+ CD4 T cells as a pharmacodynamic biomarker for anti-CTLA-4 therapy. Cancer Immunol Res 2013;1:229-34.

[40] Funt S, Charen AS, Yusko E, Vignali M, Benzeno S, Boyd ME, et al. Correlation of peripheral and intratumoral T-cell receptor (TCR) clonality with clinical outcomes in patients with metastatic urothelial cancer (mUC) treated with atezolizumab [abstract]. J Clin Oncol 2016;34(15):3005.

[41] Snyder A, Nathanson T, Funt SA, Ahuja A, Buros Novik J, Hellmann MD, et al. Contribution of systemic and somatic factors to clinical response and resistance to PD-L1 blockade in urothelial cancer: An exploratory multi-omic analysis. PLoS Med 2017;14:e1002309.

[42] Karwacz K, Bricogne C, MacDonald D, Arce F, Bennett CL, Collins M, et al. PD-L1 co-stimulation contributes to ligand-induced T cell receptor down-modulation on CD8+ T cells. EMBO Mol Med 2011;3:58192.

[43] Escors D, Bricogne C, Arce F, Kochan G, Karwacz K. On the Mechanism of T cell receptor downmodulation and its physiological significance. J Biosci Med 2011 Jan;1(1). 
[44] Postow MA, Manuel M, Wong P, Yuan J, Dong Z, Liu C, et al. Peripheral T cell receptor diversity is associated with clinical outcomes following ipilimumab treatment in metastatic melanoma. J Immunother Cancer 2015;3:23.

[45] Robert L, Tsoi J, Wang X, Emerson R, Homet B, Chodon T, et al. CTLA4 blockade broadens the peripheral T-cell receptor repertoire. Clin Cancer Res Off J Am Assoc Cancer Res 2014;20:2424-32.

[46] Oh DY, Cham J, Zhang L, Fong G, Klinger M, Faham M, et al. Association between T cell repertoire diversification and both clinical response as well as toxicity following immune checkpoint blockade in metastatic cancer patients [abstract]. J Clin Oncol 2016;34.

[47] Ferrucci PF, Gandini S, Battaglia A, Alfieri S, Di Giacomo AM, Giannarelli D, et al. Baseline neutrophil-to-lymphocyte ratio is associated with outcome of ipilimumab-treated metastatic melanoma patients. Br J Cancer 2015;112:1904-10..

[48] Moschetta M, Kasenda B, Mak G, Voskoboynik M, Martynyuk N, Formica V, et al. Early neutrophil to lymphocyte ratio dynamics to predict progression free survival in patients treated with immune-checkpoint inhibitors [abstract]. J Clin Oncol 2016;34.

[49] Nakamura Y, Kitano S, Takahashi A, Tsutsumida A, Namikawa K, Tanese K, et al. Nivolumab for advanced melanoma: pretreatment prognostic factors and early outcome markers during therapy. Oncotarget 2016;7:77404-15.

[50] Zibelman MR, Judd J, Handorf E, O'Neill J, Bentota S, Doyle J, et al. Association of eosinophila with efficacy in non-melanoma patients treated with programmed death 1 inhibitors [abstract]. J Clin Oncol 2016;34.

[51] Delyon J, Mateus C, Lefeuvre D, Lanoy E, Zitvogel L, Chaput N, et al. Experience in daily practice with ipilimumab for the treatment of patients with metastatic melanoma: an early increase in lymphocyte and eosinophil counts is associated with improved survival. Ann Oncol Off J Eur Soc Med Oncol 2013;24:1697-703.

[52] Le DT, Uram JN, Wang H, Bartlett BR, Kemberling H, Eyring AD, et al. PD-1 Blockade in Tumors with Mismatch-Repair Deficiency. N Engl J Med 2015;372:2509-20.

[53] Snyder A, Makarov V, Merghoub T, Yuan J, Zaretsky JM, Desrichard A, et al. Genetic basis for clinical response to CTLA-4 blockade in melanoma. N Engl J Med 2014;371:2189-99.

[54] Rizvi NA, Hellmann MD, Snyder A, Kvistborg P, Makarov V, Havel JJ, et al. Cancer immunology. Mutational landscape determines sensitivity to PD-1 blockade in non-small cell lung cancer. Science 2015;348:124-8.

[55] Chae YK, Anker JF, Mohindra NA, Kaplan JB, Chandra S, Carneiro BA, et al. Mutational burden and activated $T$ cell infiltration in lung squamous cell carcinomas with DNA repair mutations [abstract]. J Clin Oncol 2016;34.

[56] Tsantoulis P, Hill LA, Walker SM, Wirapati P, Graham DM, Wilson RH, et al. Association of a specific innate immune response to DNA damage with DNA repair deficient colorectal cancers [abstract]. J Clin Oncol 2016;34.

[57] Seguí E, Paré L, Adamo B, Pineda E, Marín-Aguilera M, Reig Ò, et al. Immune gene expression, survival outcome and response to PD-1/PD-L1 blockade: a TCGA pan-cancer analysis [abstract]. J Clin Oncol 2016;34. 
[58] Navarro A, Arance A, Reguart N, Paré L, Galván P, Marti AM, et al. Association of response to programmed death 1 receptor or ligand (PD1/PDL1) blockade with immune-related gene expression profiling across three cancer-types [abstract]. J Clin Oncol 2016;34.

[59] Hugo W, Zaretsky JM, Sun L, Song C, Moreno BH, Hu-Lieskovan S, et al. Genomic and Transcriptomic Features of Response to Anti-PD-1 Therapy in Metastatic Melanoma. Cell 2016;165:35-44.

[60] Riaz N, Havel JJ, Kendall SM, Makarov V, Walsh LA, Desrichard A, et al. Recurrent SERPINB3 and SERPINB4 mutations in patients who respond to anti-CTLA4 immunotherapy. Nat Genet 2016;48:1327-9.

[61] Zaretsky JM, Garcia-Diaz A, Shin DS, Escuin-Ordinas H, Hugo W, Hu-Lieskovan S, et al. Mutations Associated with Acquired Resistance to PD-1 Blockade in Melanoma. N Engl J Med 2016;375:819-29.

[62] Snyder A, Pamer E, Wolchok J. IMMUNOTHERAPY. Could microbial therapy boost cancer immunotherapy? Science 2015;350:1031-2.

[63] Sivan A, Corrales L, Hubert N, Williams JB, Aquino-Michaels K, Earley ZM, et al. Commensal Bifidobacterium promotes antitumor immunity and facilitates anti-PD-L1 efficacy. Science 2015;350:10849.

[64] Vétizou M, Pitt JM, Daillère R, Lepage $P$, Waldschmitt N, Flament $C$, et al. Anticancer immunotherapy by CTLA-4 blockade relies on the gut microbiota. Science 2015;350:1079-84.

[65] Rubio-Godoy V, Dutoit V, Zhao Y, Simon R, Guillaume P, Houghten R, et al. Positional scanningsynthetic peptide library-based analysis of self- and pathogen-derived peptide cross-reactivity with tumorreactive Melan-A-specific CTL. J Immunol Baltim Md 1950 2002;169:5696-707.

[66] Wang J-L, Chang C-H, Lin J-W, Wu L-C, Chuang L-M, Lai M-S. Infection, antibiotic therapy and risk of colorectal cancer: a nationwide nested case-control study in patients with Type 2 diabetes mellitus. Int J Cancer 2014;135:956-67.

[67] Vétizou M, Daillère R, Zitvogel L. Rôle du microbiote intestinal dans la réponse aux thérapies antitumorales. Biol Aujourdhui 2017;211:51-67.

[68] Massagué J. TGFbeta in Cancer. Cell 2008;134:215-30. doi:10.1016/j.cell.2008.07.001.

[69] Gupta V, Davancaze T, Good J, Kalia N, Anderson M and al., Bioanalytical qualification of clinical biomarker assays in plasma using a novel multi-analyte Simple Plex ${ }^{\mathrm{TM}}$ platform, Bioanalysis 2016; $8(23): 2415-2428$.

[70] Hamid O, Schmidt H, Nissan A, Ridolfi L, Aamdal S, Hansson J, et al. A prospective phase II trial exploring the association between tumor microenvironment biomarkers and clinical activity of ipilimumab in advanced melanoma. J Transl Med 2011;9:204.

[71] Hwang SL, Chung NP-Y, Chan JK-Y, Lin C-LS. Indoleamine 2, 3-dioxygenase (IDO) is essential for dendritic cell activation and chemotactic responsiveness to chemokines. Cell Res 2005;15:167-75.

[72] Singer K, Gottfried E, Kreutz M, Mackensen A. Suppression of T-cell responses by tumor metabolites. Cancer Immunol Immunother CII 2011;60:425-31.

[73] Santarpia M, González-Cao M, Viteri S, Karachaliou N, Altavilla G, Rosell R. Programmed cell death protein-1/programmed cell death ligand-1 pathway inhibition and predictive biomarkers: understanding transforming growth factor-beta role. Transl Lung Cancer Res 2015;4:728-42. 
[74] Funaki S, Shintani Y, Kawamura T, Kanzani R, Minami M and al., Chemotherapy enhances programmed cell death 1 /ligand 1 expression via TGF- $\beta$ induced epithelial mesenchymal transition in non-small cell lung cancer, Onco Rep. 2017; 38(4) : 2277-2284

Légende des tableaux et des figures :

Tableau 1: études de phase III concernant le Nivolumab et le Pembrolizumab

Tableau 2 : études de phase II concernant l'Atezolizumab (anti-PDL-1)

Figure 1 : Biomarqueurs prédictifs de réponse aux inhibiteurs de checkpoint (points de contrôle) immuns lors de la réaction immunitaire antitumorale

Predictive biomarkers of response to immune checkpoint inhibitors during antitumor immune response 


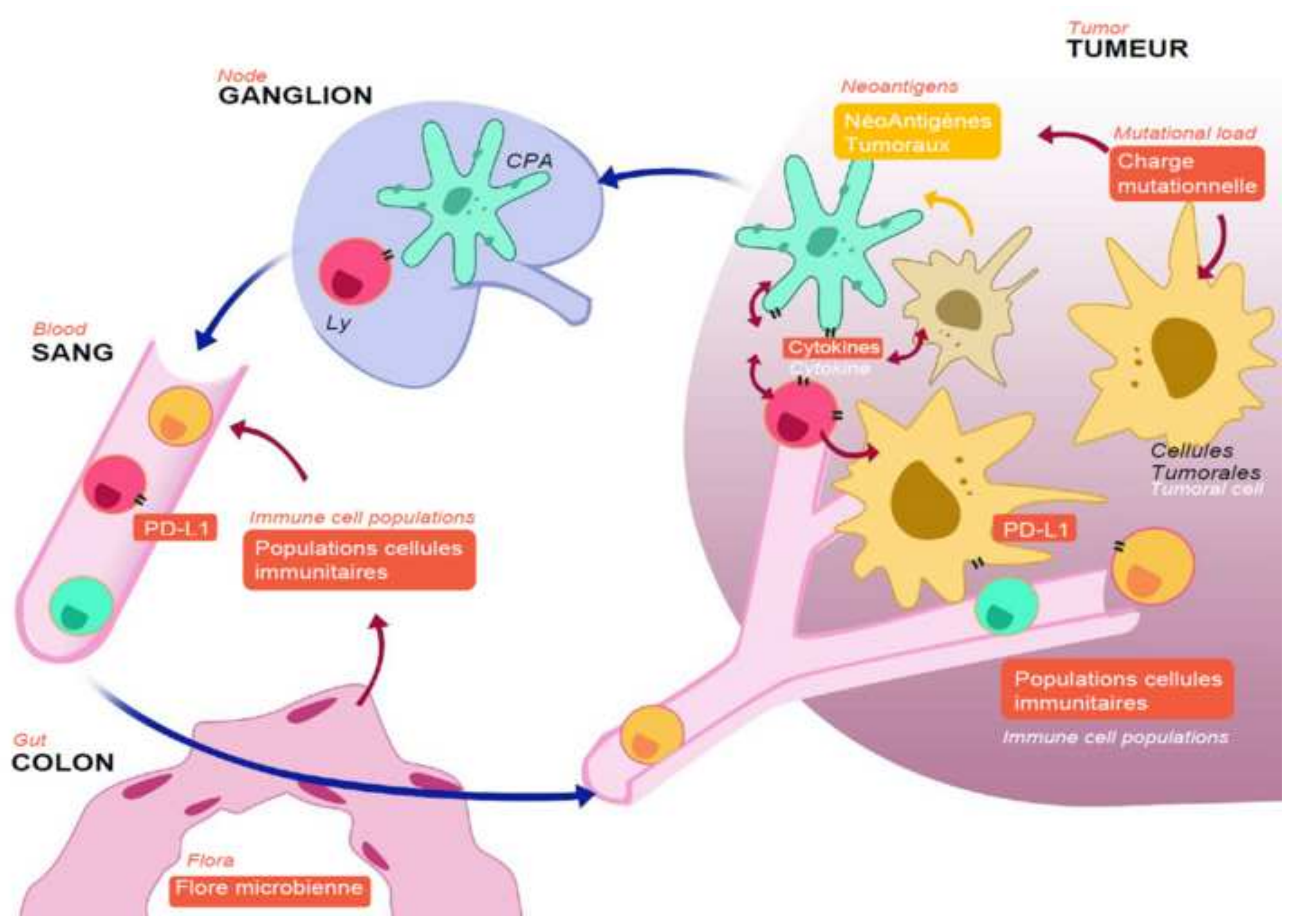


Tableau 1: études de phase III concernant le Nivolumab et le Pembrolizumab

\begin{tabular}{|c|c|c|c|c|}
\hline Etudes & Type de tumeur & $\begin{array}{l}\text { Immuno- } \\
\text { histochimie }\end{array}$ & Seuil & PD-L1 prédictif \\
\hline $\begin{array}{l}\text { CheckMate } 037 \\
{[10]}\end{array}$ & Mélanome & $\begin{array}{l}\text { DAKO } \\
\text { Clone 28-8 }\end{array}$ & $5 \%$ CT & OUI \\
\hline $\begin{array}{l}\text { CheckMate } 067 \\
{[7]}\end{array}$ & Mélanome & $\begin{array}{l}\text { DAKO } \\
\text { Clone 28-8 }\end{array}$ & $5 \%$ CT & OUI \\
\hline $\begin{array}{l}\text { CheckMate } 057 \\
{[11]}\end{array}$ & CBPNPC & $\begin{array}{l}\text { DAKO } \\
\text { Clone 28-8 }\end{array}$ & $\begin{array}{l}1 \%-5 \%-10 \% \\
\text { CT }\end{array}$ & OUI \\
\hline $\begin{array}{l}\text { CheckMate } 066 \\
{[12]}\end{array}$ & Mélanome & $\begin{array}{l}\text { DAKO } \\
\text { Clone 28-8 }\end{array}$ & $5 \%$ CT & NON \\
\hline $\begin{array}{l}\text { CheckMate } 017 \\
{[13]}\end{array}$ & CBPNPC & $\begin{array}{l}\text { DAKO } \\
\text { Clone 28-8 }\end{array}$ & $\begin{array}{l}1 \%-5 \%-10 \% \\
\text { CT }\end{array}$ & NON \\
\hline $\begin{array}{l}\text { CheckMate } 025 \\
{[8]}\end{array}$ & Carcinome rénal & $\begin{array}{l}\text { DAKO } \\
\text { Clone 28-8 }\end{array}$ & $\begin{array}{l}<1 \%,<5 \%, \geq \\
5 \% \text { CT }\end{array}$ & NON \\
\hline $\begin{array}{l}\text { KEYNOTE } 006 \\
{[14]}\end{array}$ & Mélanome & $\begin{array}{l}\text { DAKO } \\
\text { Clone 22c3 }\end{array}$ & $1 \% \mathrm{CT}+\mathrm{CME}$ & NON \\
\hline $\begin{array}{l}\text { KEYNOTE } 010 \\
{[15]}\end{array}$ & CBPNPC & $\begin{array}{l}\text { DAKO } \\
\text { Clone 22c3 }\end{array}$ & $50 \% \mathrm{CT}$ & OUI \\
\hline $\begin{array}{l}\text { KEYNOTE } 040 \\
{[16]}\end{array}$ & Cancer ORL & $\begin{array}{l}\text { DAKO } \\
\text { Clone 22c3 }\end{array}$ & $1 \% \mathrm{CT}$ & $?$ \\
\hline $\begin{array}{l}\text { KEYNOTE } 024 \\
{[17]}\end{array}$ & CBPNPC & $\begin{array}{l}\text { DAKO } \\
\text { Clone 22c3 }\end{array}$ & $50 \% \mathrm{CT}$ & $?$ \\
\hline
\end{tabular}

$\mathrm{CT}=$ Cellules tumorales $; \mathrm{CME}=$ Cellules du microenvironnement $; \mathrm{CBPNPC}=$ Cancer Broncho-Pulmonaire Non à Petites Cellules 
Tableau 2 : études de phases I-II concernant l'Atezolizumab et le Durvalumab (anti-PD-L1)

\begin{tabular}{|c|c|c|c|c|}
\hline $\begin{array}{l}\text { POPLAR [18] : } \\
\text { phase } 2\end{array}$ & CBPNPC & $\begin{array}{l}\text { Ventana } \\
\text { Clone SP } 142\end{array}$ & $\begin{array}{l}50-5-1 \% \mathrm{CT} \\
10-5-1 \% \mathrm{CME}\end{array}$ & OUI \\
\hline IMvigor 210 [19] & $\begin{array}{l}\text { Carcinome } \\
\text { urothélial }\end{array}$ & \begin{tabular}{|l|} 
Ventana \\
Clone SP 142
\end{tabular} & $1-5 \% \mathrm{CME}$ & OUI \\
\hline $\begin{array}{l}\text { NCT01693562 } \\
\text { [20] } \\
\text { Phase 1-2 }\end{array}$ & $\begin{array}{l}\text { Carcinome } \\
\text { urothélial vessie }\end{array}$ & \begin{tabular}{|l|} 
Ventana \\
Clone SP 263
\end{tabular} & $\begin{array}{l}25 \% \\
\text { CME et/ou CT }\end{array}$ & OUI \\
\hline $\begin{array}{l}\text { NCT01693562 } \\
{[21]} \\
\text { Phase 1-2 }\end{array}$ & $\begin{array}{l}\text { Carcinome } \\
\text { urothélial }\end{array}$ & $\begin{array}{l}\text { Ventana } \\
\text { Clone SP } 263\end{array}$ & $\begin{array}{l}25 \% \\
\text { CME et/ou CT }\end{array}$ & OUI \\
\hline
\end{tabular}

